\title{
SOME RECURRENCE FORMULAS FOR SPHERICAL POLYNOMIALS ON TUBE DOMAINS
}

\author{
GENKAI ZHANG
}

\begin{abstract}
For a tube domain $G / K$ we study the tensor products of two spherical representations of the maximal compact group $K$ and the product of the corresponding spherical polynomials. When one of these is a fundamental representation, we prove that the spherical representations appear with multiplicity at most one and we then find all the coefficients in the recurrence formula for the product of the spherical polynomials. This generalizes the previous result of $L$. Vretare and proves for certain cases a conjecture of R. Stanley on Jack symmetric polynomials.
\end{abstract}

\section{INTRODUCTION}

Let $D=G / K$ be a bounded symmetric domain of tube type, and let $S=$ $K / L$ be its Shilov boundary. The space of holomorphic polynomials on $D$ is decomposed into $K$-irreducible spaces. Each $K$-irreducible space contains an $L$-fixed vector. Under certain normalization it is unique. This vector is in fact the spherical polynomial corresponding to the representation. Spherical polynomials play important roles in the study of holomorphic discrete series and in the theory of special functions; see [4], [13] and [10]. In this paper we will study the problem of expanding the product of two spherical polynomials as the sum of spherical ones. Namely, let $\lambda, \mu$ be two spherical highest weights of $(K, L)$ and let $\phi_{\lambda}, \phi_{\mu}$ be the corresponding spherical polynomials, respectively. We are interested in finding the coefficients in the expansion

$$
\phi_{\lambda} \phi_{\mu}=\sum_{\delta} d_{\lambda, \mu}(\delta) \phi_{\delta} .
$$

Such an expansion is also called the recurrence formula.

In [16] Vretare studied the recurrence formula on a general symmetric space. When one of the polynomial corresponds to the "smallest" representation, all the coefficients in the formula are obtained. This result is used in our paper [11] to calculate the norm of $K$-types in vector-valued homomorphic discrete series on some classical domains. This motivates our present study of the recurrence formula.

We formulate this problem in terms of tensor product of spherical representations. This is the key point of our approach. We consider here the tensor

Received by the editors September 28,1993; originally communicated to the Proceedings of the $A M S$ by Palle E. T. Jorgensen.

1991 Mathematics Subject Classification. Primary 22E46, 33A75.

Key words and phrases. Tube domain, recurrence formula, spherical polynomial. 
product of a spherical representation by the fundamental spherical representations. We prove that all the spherical representations appear with at most multiplicity one in the tensor product. Consequently we find all the coefficients by using the result of Vretare. Our result then proves for certain cases a conjecture of R. Stanley [13]; see $\S 3$.

We note that tensor products of spherical representations also appear in the study of Toeplitz operators [15] and the algebraic study of highest weight modules [3]. We hope that our result in this paper will also shed some light on those problems.

The main result is Theorem 1 in $\S 2$. In $\S 1$ we recall some known facts about spherical polynomials on tube domains and the recurrence formula of Vretare. In $\S 3$ we make further remarks and formulate a conjecture about spherical polynomials.

\section{SOME PRELIMINARIES}

Let $D=G / K$ be an irreducible bounded symmetric domain. We assume $D$ is a tube domain of rank $r$. Let $\mathfrak{g}$ be the Lie algebra of $G$ and $\mathfrak{g}=\mathfrak{k}+\mathfrak{p}$ be the Cartan decomposition. $\mathfrak{h}$ will be a Cartan subalgebra of $\mathfrak{k}$. Let $\Phi^{+}$be the set of noncompact positive roots of $\left(\mathfrak{g}^{\mathbb{C}}, \mathfrak{h}^{\mathbb{C}}\right)$. A basis of root vector $\left\{e_{\alpha}\right\}$ can be chosen so that $\tau e_{\alpha}=-e_{-\alpha},\left[e_{\alpha}, e_{-\alpha}\right]=h_{\alpha}$, and $\left[h_{\alpha}, e_{ \pm \alpha}\right]=2 e_{ \pm \alpha}$, where $\tau$ is the conjugation with respect to the real form $\mathfrak{k}+i \mathfrak{p}$. So we have the root space decomposition of $\mathfrak{g}^{\mathbb{C}}$,

$$
\mathfrak{g}^{\mathbb{C}}=\mathfrak{p}^{-}+\mathfrak{k}^{\mathbb{C}}+\mathfrak{p}^{+},
$$

where $\mathfrak{p}^{-}$and $\mathfrak{p}^{+}$are sum of the negative and positive noncompact root spaces. So $D$ is a bounded domain in $\mathfrak{p}^{+}$.

Let $\gamma_{1}>\cdots>\gamma_{r}$ be the Harish-Chandra strongly orthogonal roots with the corresponding root vectors $e_{j}=e_{\gamma_{j}}$, and let $h_{j}=h_{\gamma_{j}}$ so that $\gamma_{j}\left(h_{k}\right)=2 \delta_{j, k}$. Let

$$
e=e_{1}+\cdots+e_{r}
$$

The Shilov boundary of $D$ is $S=K \cdot e=K / L$ and $L=\{k \in K, k \cdot e=e\}$. Here the action is just the adjoint action of $K$ on $\mathfrak{p}^{+}$.

For an $r$-tuple of integers $\underline{\mathbf{m}}=\left(m_{1}, \ldots, m_{r}\right)$ with

$$
m_{1} \geq m_{2} \geq \cdots \geq m_{r} \geq 0,
$$

we abuse notation and denote $\underline{\mathbf{m}}=m_{1} \gamma_{1}+\cdots+m_{r} \gamma_{r}$. The following is a known result of Schmid; see [4] and [14].

Theorem S. The space $\mathscr{P}$ of holomorphic polynomials on $\mathfrak{p}^{+}$decomposes into irreducible subspaces under $\operatorname{Ad}(K)$ as

$$
\mathscr{P} \cong \sum_{\underline{\mathbf{m}} \geq 0} V^{\mathbf{m}}
$$

Each $V \underline{\underline{m}}$ is of highest weight $-\underline{\mathbf{m}}=-\left(m_{1} \gamma_{1}+\cdots+m_{r} \gamma_{r}\right)$ and it contains $a$ unique $L$-fixed vector $\phi_{\underline{m}}$ such that $\phi_{\underline{m}}(e)=1$.

For notational convenience we may take the anti-representation and say that the module $V \underline{\underline{m}}$ has highest weight $\underline{m}$. 
It follows from the above theorem that the highest weights

$$
\mu^{j}=\sum_{k=1}^{j} \gamma_{k}, \quad j=1, \ldots, r,
$$

are the fundamental holomorphic spherical representations of $(K, L)$. Our main interest will be the tensor products $V^{\underline{m}} \otimes V^{\mu^{j}}$ of $V^{\underline{m}}$ with $V^{\mu^{j}}$.

To realize the polynomial spaces above we may also start with an abstract module and view the polynomial as matrix coefficients; see [6]. This will help us to clarify some calculations in the next section. So we abuse notation and let $V \underline{\mathbf{m}}$ be a module of $K$ with highest weight $\underline{\mathbf{m}}$. We know that each module $V \underline{\underline{m}}$ has a $L$-fixed vector, say $\phi_{\underline{\underline{m}}}$. We normalize it so $\left\|\phi_{\underline{m}}\right\|=1$. Write a point $Z$ in $S$ as $Z=k^{-1} \cdot e$ with $k \in K$. For any vector $v \in V^{\underline{m}}$ we associate a function on $S$ by

$$
v(Z)=\left\langle k v, \phi_{\underline{\mathbf{m}}}\right\rangle .
$$

So $\phi_{\underline{\mathbf{m}}}(Z)$ is an element in $\mathscr{P}$ with $\phi_{\underline{\mathbf{m}}}(e)=1$. See [6], Chapter V, and [4].

Now we have that $K / L$ is a compact symmetric space. Let $\mathfrak{k}=\mathfrak{l}+\mathfrak{q}$ be the Cartan decomposition and $\mathfrak{k}^{*}=\mathfrak{l}+i \mathfrak{q}$ be the corresponding noncompact real form. Let $K^{*}$ be the corresponding group. So $\Omega=K^{*} \cdot e=K^{*} / L$ is a homogeneous cone. See [4], pp. 66-68 for further details.

So we have $\mathfrak{h}^{\mathbb{C}}=\mathfrak{h}_{\mathfrak{l}}^{\mathbb{C}}+\mathfrak{h}_{\mathfrak{q}}^{\mathbb{C}}$, where $\mathfrak{h}_{\mathfrak{l}}$ and $\mathfrak{h}_{\mathfrak{q}}$ are maximal abelian in $\mathfrak{l}$ and $\mathfrak{q}$, respectively. In fact, $\mathfrak{h}_{\mathfrak{q}}$ is just the linear subspace generated by $i h_{1}, \ldots, i h_{r}$. The root system $\Delta_{0}^{+}$for $\left(\mathfrak{k}^{*}, i \mathfrak{h}_{\mathfrak{q}}\right)$ consists of $\left\{ \pm \frac{\gamma_{k}-\gamma_{j}}{2}, k<j\right\}$ with the given ordering. The corresponding root spaces have a common dimension $a$. Let $\rho$ be the half of the sum of positive roots. Then

$$
\rho=\frac{1}{2} \sum_{\alpha \in \Delta_{0}^{+}} m_{\alpha} \alpha=\frac{a}{4} \sum_{j=1}^{r}(r-(2 j-1)) \gamma_{j} .
$$

Let $W$ be the Weyl group of $\Delta_{0}$. Then $W$ is just the symmetric group of $\{1, \ldots, r\}$.

We now recall the result of Vretare ([16], Corollary 4.2). We will formulate in a slightly different way.

The irreducible representations of $K$ that have $L$-fixed vectors are called spherical representations. The highest weights of such representations are characterized by the Cartan-Helgason Theorem (see [6], Chapter V). In particular, the highest weights vanish on $\mathfrak{h}_{\mathfrak{l}}^{\mathbb{C}}$ and we can view them as (real) linear functionals on $i h_{\mathrm{q}}$. Let $\Lambda$ be the lattice of spherical highest weights. For each $\lambda \in \Lambda$ we have the corresponding spherical function on $K$, which we will denote by $\phi_{\lambda}$. (This notation is consistent with our previous $\phi_{\underline{m}}$.) Let $\preccurlyeq$ be a partial ordering of $i h_{\mathrm{q}}$ such that

$$
\left\langle\mu_{1}, \lambda\right\rangle\left\langle\left\langle\mu_{2}, \lambda\right\rangle, \quad \text { for all } \lambda \in \Lambda \text { if } \mu_{1} \preccurlyeq \mu_{2},\right.
$$

where $\langle\cdot, \cdot\rangle$ is the Killing form.

For any highest weight $x$ we denote by $W_{x}$ the isotropy group of $x$ in $W$. For any spherical highest weights $\mu$ and $\delta$ the next result gives all the coefficients of $\phi_{\delta+S \mu}$ with $S \in W$ in the expansion of $\phi_{\delta} \phi_{\mu}$. 
Theorem $\mathbf{V}$. We have the following recurrence formula

$$
\phi_{\mu}(g) \phi_{\delta}(g)=\sum_{-\mu \preccurlyeq x \preccurlyeq \mu, S \in W / W_{x}} d_{x}(S(\delta+\rho)) \phi_{\delta+S^{-1} x}(g), \quad g \in K .
$$

Moreover, the coefficients $d_{\mu}(\lambda)$ are given by

$$
d_{\mu}(\lambda)=C(-i(\mu+\rho)) \frac{C(-i \lambda)}{C(-i(\lambda+\mu))} .
$$

Remark. The result stated in [16] is for semisimple groups. The group $K$ is not semisimple, but it is easy to extend the result to our case.

\section{THE MAIN THEOREM}

We denote by $J$ the set $\{1,2, \ldots, j\}$ and denote by $I(J)$ the set $\left\{i_{1}, i_{2}\right.$, $\left.\ldots, i_{j}\right\}$ of $j$-integers with $1 \leq i_{1}<i_{2}<\cdots<i_{j} \leq r$.

Lemma 1. Let $\underline{\underline{n}}=\sum_{j=1}^{r} n_{j} \gamma_{j}$. We have

$$
d_{\mu^{j}}(\underline{\mathbf{n}})=C\left(-i\left(\mu^{j}+\rho\right)\right) \prod_{l \in J, k \notin J, l \leq k} \frac{\frac{a}{2}+n_{l}-n_{k}}{n_{l}-n_{k}} .
$$

Moreover, $C(-i(\mu+\rho))>0$.

Proof. Using the formula for $C$-function [6] we find that

$$
\begin{aligned}
d_{\mu}(\underline{\mathbf{n}}) & =C(-i(\mu+\rho)) \frac{C(-i(\underline{\mathbf{n}}))}{\left.C\left(-i \underline{\mathbf{n}}+\mu^{j}\right)\right)} \\
& =C(-i(\mu+\rho)) \prod_{l<k} \frac{\Gamma\left(n_{l}-n_{k}\right)}{\Gamma\left(\frac{a}{2}+n_{l}-n_{k}\right)} \frac{\Gamma\left(\frac{a}{2}+n_{l}+\mu_{l}-n_{k}-\mu_{k}-k\right)}{\Gamma\left(n_{l}+\mu_{l}-n_{k}-\mu_{k}\right)},
\end{aligned}
$$

as a meromorphic function on $\left(\mathfrak{h}_{\mathfrak{q}}^{\mathbb{C}}\right)^{*}$, the dual of $\mathfrak{h}_{\mathfrak{q}}^{\mathbb{C}}$ in $\S 1$.

We know that $\mu_{l}-\mu_{k}=0$ when $l, k \in J$ or when $l, k \notin J$. Thus we find

$$
d_{\mu^{j}}(\underline{\mathbf{n}})=C(-i(\mu+\rho)) \prod_{l<k, l \in J, k \notin J} \frac{\frac{a}{2}+n_{l}-n_{k}}{n_{l}-n_{k}} .
$$

The fact that $C(-i(\mu+\rho))>0$ can easily be read off from the formula for the $C$-function.

For two spherical modules $V^{\lambda}$ and $V^{\mu}$ of $(K, L)$ we denote by $\left(V^{\lambda} \otimes V^{\mu}\right)^{L}$ the sum of those spherical irreducibles in the irreducible decomposition of $V^{\lambda} \otimes$ $V^{\mu}$.

Our main theorem is the following

Theorem 1. Let $\underline{\mathbf{m}} \geq 0$ and $\mu^{j}$ be the highest weight as above.

(1) We have

$$
\left(\mu^{j} \otimes \underline{\mathbf{m}}\right)^{L}=\sum_{1 \leq i_{1}<i_{2}<\cdots<i_{j} \leq r}\left(\underline{\mathbf{m}}+\gamma_{i_{1}}+\cdots+\gamma_{i_{j}}\right),
$$

where the irreducible $\underline{\mathbf{m}}+\gamma_{i_{1}}+\cdots+\gamma_{i_{j}}$ in the summation appears with at most multiplicity one and it appears if it does not violate the condition for a highest weight (1.1). 
(2) The following recurrence formula holds:

$\phi_{\mu j}(g) \phi_{\underline{\mathbf{m}}}(g)=\sum_{1 \leq i_{1}<i_{2}<\cdots<i_{j} \leq r} d_{\underline{\mathbf{m}}, \mu^{j}}\left(\underline{\mathbf{m}}+\gamma_{i_{1}}+\cdots+\gamma_{i_{j}}\right) \phi_{\underline{\mathbf{m}}+\gamma_{i_{1}}+\cdots+\gamma_{i_{j}}}(g), \quad g \in K$, where $d_{\underline{\mathbf{m}}, \mu^{j}}\left(\underline{\mathbf{m}}+\gamma_{i_{1}}+\cdots+\gamma_{i_{j}}\right)=d_{\mu^{j}}(\underline{\mathbf{n}})$ is given in Lemma 1 with $\underline{\mathbf{n}}=\underline{\mathbf{m}}+S^{-1} \mu^{j}$ and $S \in W$ is such that $S^{-1} \mu^{j}=\gamma_{i_{1}}+\cdots+\gamma_{i_{j}}$. Moreover,

$$
d_{\underline{\mathbf{m}}, \mu^{j}}\left(\underline{\mathbf{m}}+\gamma_{i_{1}}+\cdots+\gamma_{i_{j}}\right) \neq 0
$$

if and only if $\underline{\mathbf{m}}+\gamma_{i_{1}}+\cdots+\gamma_{i_{j}}$ appears in the decomposition in (1).

Remark. If we take the domain $D$ to be $S U(n, n) / S(U(n) \times U(n))$, then the spherical polynomials $\phi_{\underline{m}}$ of a highest weight $\underline{m}$ is $\chi_{\underline{m}} / \operatorname{dim}(V \underline{m})$, where $\chi_{\underline{m}}$ is the character on $U(n)^{\mathbb{C}}$, given by the Schur function on the diagonal matrices. Our theorem can then be obtained by using the general Littlewood-Richardson rule [9].

The proof of this theorem is based on the recurrence formula in Theorem $\mathrm{V}$ and a detailed analysis of the tensor product.

Definition. Let $V^{\lambda}$ be a representation of $K$ with highest weight $\lambda$. Suppose $\mu$ is a weight appearing in $V^{\lambda} . \mu$ is called a spherical weight if $\mu=\sum_{j=1}^{r} \mu_{j} \gamma_{j}$ for some integers $\mu_{j}$.

Lemma 2. The spherical weights appearing in $\mu^{j}$ are of the form $\gamma_{i_{1}}+\cdots+\gamma_{i_{j}}$, where $1 \leq i_{1}<\cdots<i_{j} \leq r$. Moreover, they all have multiplicity one.

Proof. There are five types of irreducible tube domains (see, e.g., [7]):

(A) $U(n, n) / U(n) \times U(n)$

(B) $S O(2, q) / S O(2) \times S O(q)$;

(C) $S p(n, \mathbb{R}) / U(n)$

(D) $S O^{*}(4 n) / U(2 n)$;

(E) $E_{7(-25)} / E_{6} \times S O(2)$.

We prove the lemma case-by-case. First we notice that $\mu^{1}=\gamma_{1}$ is the highest weight for the representation on $\mathfrak{p}^{+}$and $\mu^{r}=\gamma_{1}+\cdots+\gamma_{r}$ is a one-dimensional representation, so our Lemma follows from the root space decomposition. In particular, if the domain is of rank two, our lemma is true. The type B domain above is of rank two, so we consider the other types.

Type A. The rank is $n$. The Shilov boundary is $U(n)=U(n) \times U(n) / U(n)$. Take a Cartan algebra $\eta^{\mathrm{C}}$ of $u(n)^{\mathrm{C}}$ to be the linear span of diagonal matrices $E_{i}$ with the $i$ th term being 1 and the rest 0 . Let $\varepsilon_{i}$ be the linear functional on $\eta^{\mathbb{C}}$ dual to $E_{i}$. The Harish-Chandra strongly orthogonal roots are $\gamma_{i}=\varepsilon_{i} \otimes \varepsilon_{i}$, $i=1, \ldots, n$. Therefore,

$$
\mu^{j}=\gamma_{1}+\cdots+\gamma_{j}=\left(\varepsilon_{1}+\cdots+\varepsilon_{j}\right) \otimes\left(\varepsilon_{1}+\cdots+\varepsilon_{j}\right)^{*},
$$

as the highest weight of the representation of $U(n) \times U(n)$, where $\left(\varepsilon_{1}+\cdots+\varepsilon_{j}\right)^{*}$ indicates the contragradient.

We need the following simple fact about $U(n)$-representations.

Fact. The weights appearing in $\varepsilon_{1}+\cdots+\varepsilon_{j}$ are of the form $\varepsilon_{i_{1}}+\cdots+\varepsilon_{i_{j}}$ with $1 \leq i_{1}<i_{2}<\cdots<i_{j} \leq n$, and they all appear with multiplicity one.

Thus the spherical weights in $\mu^{j}$ are of the form $\gamma_{i_{1}}+\cdots+\gamma_{i_{j}}, i_{1}<\cdots<i_{j}$, and they all appear with multiplicity one. 
Type C. The rank is $n$, and the Shilov boundary is $U(n) / O(n)$. Take the Cartan algebra of $\mathfrak{u}(n)^{\mathbb{C}}$ as above. The Harish-Chandra orthogonal roots are $\gamma_{j}=2 \varepsilon_{j}$, so

Thus we have

$$
\mu^{j}=\gamma_{1}+\cdots+\gamma_{j}=2\left(\varepsilon_{1}+\cdots+\varepsilon_{j}\right)
$$

$$
\mu^{j}=\left(\varepsilon_{1}+\cdots+\varepsilon_{j}\right) \otimes_{s}\left(\varepsilon_{1}+\cdots+\varepsilon_{j}\right),
$$

the symmetric tensor product. Our lemma then follows easily from the definition of the symmetric tensor product argument and the above fact .

Type D. The rank is $n$ and the Shilov boundary is $U(2 n) / S p(n)$. Take $\left\{\varepsilon_{k}\right\}_{k=1}^{2 n}$ as in Type I. We have that the Harish-Chandra strongly orthogonal roots are

$$
\gamma_{j}=\varepsilon_{2 j-1}+\varepsilon_{2 j}
$$

so

$$
\mu^{j}=\gamma_{1}+\cdots+\gamma_{j}=\varepsilon_{1}+\varepsilon_{2}+\cdots+\varepsilon_{2 j-1}+\varepsilon_{2 j} .
$$

This is a special case of the above fact.

Type E. The rank is 3 and the Shilov boundary is $E_{6} \times S O(2) / F_{4}$. We need only to consider $\mu^{2}=\gamma_{1}+\gamma_{2}$. Using the standard notation [3] we know that $\mu^{2}$ is $\omega_{6}$ in [1]. This is a 27-dimensional representation of $E_{6} \times S O(2)$, and moreover all weights appearing are of multiplicty one ([2], p. 129). Consider the root system of $E_{7}$ with a compact Cartan of $E_{6} \times S O(2)$; see [12] and [3]. An elementary calculation using the Freudenthal's formula [18] shows that 24 weights in $\mu^{2}$ are of the form $\omega_{6}-\alpha$ with $\alpha$ a positive compact root, which are not spherical, and rest 3 weights are spherical: $\gamma_{1}+\gamma_{2}, \gamma_{2}+\gamma_{3}$ and $\gamma_{1}+\gamma_{3}$.

This completes the proof the lemma.

Let $\left(V^{\lambda}, \lambda\right)$ and $\left(V^{\mu}, \mu\right)$ be two spherical representations of the group $K$. The following fact is elementary, see [18].

Lemma 3. $y$ The highest weight of an irreducible submodule of $V^{\lambda} \otimes V^{\mu}$ is of the form $\lambda+\alpha$, where $\alpha$ is a weight appearing in $V^{\mu}$.

The next lemma clarifies the relation between the irreducible decomposition of the tensor product of two representations and the recurrence formula for the product of the corresponding spherical polynomials. We include here a simple proof. We recall the notation $\left(V^{\lambda} \otimes V^{\mu}\right)^{L}$ defined above.

Lemma 4. Suppose

$$
\left(V^{\lambda} \otimes V^{\mu}\right)^{L}=\sum_{\delta} \sum_{j=1}^{n(\delta)} V_{j}^{\delta},
$$

where $n(\delta)$ is the multiplicity of $\delta$ in the decomposition (all $V_{j}^{\delta}$ have the same highest weight $\delta$ ). Then we have

$$
\phi_{\lambda}(g) \phi_{\mu}(g)=\sum_{\delta} d_{\lambda, \mu}(\delta) \phi_{\delta}(g), \quad g \in K,
$$

for some nonnegative numbers $d_{\lambda, \mu}(\delta)$.

Proof. Take $v \in\left(V^{\lambda}\right)^{L}$ and $v^{\prime} \in\left(V^{\mu}\right)^{L}$ so that $\|v\|=\left\|v^{\prime}\right\|=1$. By definition we have

$$
\phi_{\lambda}(g) \phi_{\mu}(g)=\langle g v, v\rangle\left\langle g v^{\prime}, v^{\prime}\right\rangle=\left\langle g\left(v \otimes v^{\prime}\right), v \otimes v^{\prime}\right\rangle
$$


We decompose $v \otimes v^{\prime}$ according to the irreducible decomposition of $V^{\lambda} \otimes V^{\mu}$. It is clear that $v \otimes v^{\prime} \in\left(V^{\lambda} \otimes V^{\mu}\right)^{L}$. So

$$
v \otimes v^{\prime}=\sum_{\delta} \sum_{j=1}^{n(\delta)} c(\delta, j) v_{\delta, j}
$$

where $v_{\delta, j}$ is the unique $L$-fixed vector in $V_{j}^{\delta}$ so that $\left\|v_{\delta, j}\right\|=1$. Substituting this into the above we get

$$
\begin{aligned}
\phi_{\lambda}(g) \phi_{\mu}(g) & =\left\langle g \sum_{\delta} \sum_{j=1}^{n(\delta)} c(\delta, j) v_{\delta, j}, \sum_{\delta} \sum_{j=1}^{n(\delta)} c(\delta, j) v_{\delta, j}\right\rangle \\
& =\sum_{\delta} \sum_{j=1}^{n(\delta)}|c(\delta, j)|^{2}\left\langle g v_{\delta, j}, v_{\delta, j}\right\rangle
\end{aligned}
$$

by orthogonal relation. However, unitarily equivalent representations have the same spherical polynomials, so

$$
\phi_{\lambda}(g) \phi_{\mu}(g)=\sum_{\delta}\left(\sum_{j=1}^{n(\delta)}|c(\delta, j)|^{2}\right) \phi_{\delta}(g) .
$$

This is just our lemma with $d_{\lambda, \mu}(\delta)=\sum_{j=1}^{n(\delta)}|c(\delta, j)|^{2}$.

Since spherical polynomials are orthogonal in the space $L^{2}(K / L)$ [16], it follows that there is a unique way to write the product of $\phi_{\lambda}(g) \phi_{\mu}(g)$ as the sum of spherical polynomials. Thus we have

Corollary. If $d_{\lambda, \mu}(\delta) \neq 0$ in Lemma 4 , then $\lambda \otimes \mu$ contains a spherical submodule with highest weight $\delta$.

Lemma 5. Consider the tensor product $\underline{m} \otimes \mu^{j}$. The highest weights of the spherical components (i.e., in $\left(\underline{\mathbf{m}} \otimes \mu^{j}\right)^{L}$ ) are of the form $\underline{\mathbf{m}}+\gamma_{i_{1}}+\cdots+\gamma_{i_{j}}$, where $1 \leq i_{1}<\cdots<i_{j} \leq r$ and they appear with multiplicity at most one.

Proof. This follows directly from Lemma 2 and Lemma 3.

Proof of Theorem 1. We prove (2) first. By Lemma 5 we know that the spherical highest weights appearing in $\underline{\mathbf{m}} \otimes \mu^{j}$ are of the form $\underline{\mathbf{m}}+\gamma_{i_{1}}+\cdots+\gamma_{i_{j}}$ with $1 \leq i_{1}<i_{2}<\cdots<i_{j} \leq r$. This is the same as the set $\left\{\underline{\mathrm{m}}+S^{-1} \mu^{j}, S \in W / W_{\mu^{j}}\right\}$, so all the coefficients in the recurrence formula of $\phi_{\underline{m}}(g) \phi_{\mu^{j}}(g)$ are given by Theorem V.

Fix $1 \leq i_{1}<i_{2}<\cdots<i_{j} \leq r$, and take $S \in W$ such that $S^{-1}: k \rightarrow i_{k}$ for $k=1, \ldots, j$. Thus $\underline{\mathbf{m}}+\gamma_{i_{1}}+\cdots+\gamma_{i_{j}}=\underline{\mathbf{m}}+S^{-1} \mu^{j}$. Let $\underline{\mathbf{n}}=S(\underline{\mathbf{m}}+\rho)$.

By Theorem $\mathbf{V}$ and Lemma 1 we know that the coefficient of $\phi_{\underline{m}+S^{-1} \mu^{j}}$ is given by

$$
d_{\mu^{j}}(\underline{\mathbf{n}})=C\left(-i\left(\mu^{j}+\rho\right)\right) \prod_{l<k, l \in J, k \notin J} \frac{\frac{a}{2}+n_{l}-n_{k}}{n_{l}-n_{k}} .
$$


Hence, $d_{\mu^{j}}(\underline{\mathbf{n}})=0$ if and only if

$$
\frac{a}{2}+n_{l}-n_{k}=0 \text { for some } l<k, l \in J, k \notin J .
$$

Note that $n_{l}=m_{i_{l}}-\frac{a}{2} i_{l}+\frac{a(r+1)}{4}$ for $l \in J$.

Case I. $k=i_{k^{\prime}}$ for some $k^{\prime} \in J$. In this case we have $n_{k}=m_{i_{k^{\prime}}}-\frac{a}{2} i_{k^{\prime}}+$ $\frac{a(r+1)}{4}$. Condition $(2.1)$ now reads

$$
m_{i_{l}}-m_{i_{k^{\prime}}}=\frac{a}{2}\left(-1+i_{l}-i_{k^{\prime}}\right) .
$$

However, if $i_{l}>i_{k^{\prime}}+1$, then the RHS of the above is $>0$ and the LHS is $m_{i_{l}}-m_{i_{k}} \leq 0$; if $i_{l}<i_{k^{\prime}}+1$, then the RHS is $<0$ and the LHS is $\geq 0$. Therefore, the equality holds if and only if $i_{l}=i_{k^{\prime}}+1$ and $m_{i_{l}-1}=m_{i_{l}}$, that is, $\underline{\mathbf{m}}+\mu^{j}$ does not satisfy the condition for highest weight.

Case II. $k \notin J \cup I(J)$. Then

$$
n_{k}=m_{k}-\frac{a}{2} k+\frac{a}{4}(r+1)
$$

and the condition $(2.1)$ now reads

$$
m_{i_{l}}-m_{k}=\frac{a}{2}\left(-1+i_{l}-k\right) .
$$

Similar arguments show that this is so if and only if $\underline{\mathbf{m}}+\mu^{j}$ does not satisfy the condition for a highest weight. This proves (2) of the theorem.

Now (1) of our theorem follows from (2), Lemma 5, and the corollary after Lemma 4.

Evaluating the equaltity of Theorem 1 at $g=1$ we get

Corollary. The following identity holds:

$$
\sum_{S \in W / W_{\mu^{j}}} d_{\mu^{j}}(S(\underline{\mathbf{m}}+\rho))=1 .
$$

For the exceptional domain Type E with $j=1$ the above equality reads

$$
\begin{gathered}
\frac{1}{3} \frac{8+m_{1}-m_{2}}{4+m_{1}-m_{2}} \frac{12+m_{1}-m_{3}}{8+m_{1}-m_{3}}+\frac{1}{3} \frac{m_{2}-m_{1}}{m_{2}-m_{1}-4} \frac{8+m_{2}-m_{3}}{4+m_{2}-m_{3}} \\
+\frac{1}{3} \frac{m_{3}-m_{1}-4}{m_{3}-m_{1}-8} \frac{m_{3}-m_{2}}{m_{3}-m_{2}-4}=1 .
\end{gathered}
$$

\section{SOME REMARKS}

As the spherical polynomials considered above are Jack symmetric polynomials (see [10], [13] and [17]), our main theorem above then proves the Conjecture 8.4 in [13] for the case when $\alpha=\frac{2}{a}$ with $a=1,2,4,8$ and when $\mu=\mu^{j}=(1, \ldots, 1,0, \ldots, 0)$. (One of the main theorems in [13] gives the recurrence formula for the product of Jack symmetric functions when $\mu=(n, 0, \ldots, 0)$.$) We formulate here a closely related conjecture about spher-$ ical polynomials which might be interesting from the point of view of representation theory. 
Let $\lambda$ and $\mu$ be two spherical highest weights. We decompose $\lambda \otimes \mu$ into irreducibles and take the spherical components

$$
(\lambda \otimes \mu)^{L}=\sum_{\delta} n_{\lambda, \mu}(\delta) \delta .
$$

According to Lemma 2 we have

$$
\phi_{\lambda}(g) \phi_{\mu}(g)=\sum_{\delta} d_{\lambda, \mu}(\delta) \phi_{\delta}(g), \quad g \in K,
$$

for some $d_{\lambda, \mu} \geq 0$.

Conjecture. $n_{\lambda, \mu}(\delta) \neq 0$ if and only if $d_{\lambda, \mu}(\delta) \neq 0$.

Our Lemma 4 implies that the "if" part is true, and our theorem implies that the conjecture is true when $\mu$ is a fundamental spherical representations. Note that Conjecture 8.4 in [13] implies our conjecture.

\section{ACKNOWLEDGMENT}

The author would like to thank Bent Ørsted for some helpful discussions and constant encouragement.

\section{REFERENCES}

1. N. Bourbaki, Groupes et algebres de Lie, Chapitres 4, 5 et 6, Hermann, Paris, 1975.

2. __ Groupes et algebres de Lie, Chapitres 7 et 8, Hermann, Paris, 1975.

3. T. J. Enright and A. Joseph, An intrinsic analysis of unitarizabel highest weight modules, Math. Ann. 288 (1990), 571-594.

4. J. Faraut and A. Koranyi, Function spaces and reproducing kernels on bounded symmetric, J. Funct. Anal. 89 (1990), 64-89.

5. S. Helgason, Differential geometry, Lie groups and symmetric spaces, Academic Press, London, 1978.

6. __ Groups and geometric analysis, Academic Press, London, 1984.

7. B. Kostant and S. Sahi, The Capelli identity, tube domains, and the generalized Laplace transform, Adv. Math. 87 (1991), 71-92.

8. O. Loos, Bounded symmetric domains and Jordan pairs, University of California, Irvine, 1977.

9. I. G. Macdonald, Symmetric functions and Hall polynomials, Oxford University Press, New York, 1979.

10. _ Commuting differential operators and zonal spherical functions, Algebraic groups, Utrecht 1986, Lectures Notes in Math., vol. 1271, Springer-Verlag, New York, 1987.

11. B. Ørsted and G. Zhang, Reproducing kernels and composition series for spaces of vector-valued holomorphic functions on tube domains, J. Funct. Anal. 124 (1994), 181-204.

12. W. Schmid, Die Randwerte holomorpher Functionen auf hermitesch symmetrischen Räumen, Invent. Math. 9 (1969), 61-80.

13. R. P. Stanley, Some combinatorial properties of Jack symmetric functions, Adv. Math. 77 (1989), 76-115.

14. H. Upmeier, Jordan algebras and harmonic analysis on symmetric spaces, Amer. J. Math. 108 (1986), 1-25.

15. $\_$Toeplitz operators on bounded symmetric domains, Tran. Amer. Math. Soc. 280 (1983), 221-237.

16. L. Vretare, Elementary spherical functions on symmetric spaces, Math. Scand. 39 (1976), 343-358. 
17. Z. Yan, Thesis, CUNY Graduate School, 1990.

18. D. P. Zhelobenko, Compact Lie groups and their representations, Transl. Math. Monographs, vol. 40, Amer. Math. Soc., Providence, RI, 1973.

Institut for Matematik og Datalogi, Odense Universitet, DK-5230 Odense M, DenMARK

Current address: School of Mathematics, University of New South Wales, Kensington, N.S.W. 2033, Austrailia

E-mail address: genkai@solution.maths.unsw.edu.au 